\title{
Direct visualization of single membrane scission events at clathrin- coated pits using a novel optical assay and evanescent field microscopy.
}

\section{J. Merrifield ${ }^{1}$, D. Perrais ${ }^{2}$, and D. Zenisek ${ }^{3}$}

MRC Laboratory of Molecular Biology, Hills Rd., Cambridge CB2 2QH, UK Laboratoire de Physiologie Cellulaire de la Synapse, CNRS UMR 5091 et Université Bordeaux 2 Institut François Magendie, 1 rue Camille Saint Saëns, 33077 Bordeaux Cedex France

Department of Cellular and Molecular Physiology, Yale University School of Medicine, 333 Cedar Street, New Haven, Connecticut 06520 USA

Clathrin-mediated endocytosis (CME) is one of the principal mechanisms by which eukaryotic cells internalize cell surface receptors involved in metabolism and cell signaling [1]. This conserved process entails the selective concentration of receptors at specialized domains at the plasma membrane called clathrin-coated pits (CCPs) that invaginate and pinch off of the plasma membrane along with their receptor cargo to form clathrin-coated vesicles (CCVs).

The complex process of CCP invagination and scission is driven by a consortium of different types of protein including an atypical GTPase (dynamin) [2], proteins involved in lipid modifications (such as synaptojanin) [3] and proteins involved in remodeling the actin cytoskeleton (such as cortactin, mAbp1, N-WASP and Arp3) [4]. However, while many different protein types have been implicated in CME and are known to interact with one another, it is poorly understood how these components are temporally and spatially coordinated to drive CCP invagination, membrane scission and liberation of CCVs.

Recently, evanescent field (EF) fluorescence microscopy has proven a powerful tool for probing the dynamics of CME in live cells [5]. EF microscopy uses total internal reflection to generate a thin evanescent field of excitation light at the glass/water interface at the upper surface of a coverslip [6]. Because the intensity of excitation light within the evanescent field decays exponentially with distance away from the coverslip (typically decreasing 'e' fold over 50-100 nm) only structures at or near the lower surface of a cell adherent to the coverslip are illuminated.

By using EF and epifluorescence (Epi) microscopy in tandem, the relative displacement of fluorescently labeled organelles from the glass/water interface, and hence the plasma membrane, can be monitored over time with sub-micron resolution [7]. Thus, by labeling CCPs red using clathrin-DsRed and a second protein of interest green using green fluorescent protein (GFP) it is possible to simultaneously monitor the inward movement of CCPs during CME and the recruitment of functionally relevant proteins [8].

Using this approach it was shown that the inward movement of CCPs during CME was temporally and spatially correlated with the sequential recruitment of the large GTPase dynamin and proteins involved in actin polymerization [8, 9]. However, while CCP movement most probably corresponded to a late stage in CME, it was not known whether movement corresponded to $\mathrm{CCP}$ invagination or the movement of a 
newly formed CCV away from the plasma membrane [10]. Hence, it was also unknown when the recruitment of dynamin and proteins involved in actin polymerization occurred relative to membrane scission and thus which model(s) of the molecular mechanism of CME could be correct.

To help resolve this issue we have developed a novel optical assay to detect membrane scission at single CCPs by monitoring the accessibility of a $\mathrm{pH}$ sensitive fluorescent CCP cargo to extracellular protons. The reporter for CCP accessibility is composed of a $\mathrm{pH}$ sensitive version of GFP (phluorin) attached to the extracellular domain of transferrin receptor (Tfnr) to form Tfnr-phl. When imaged using EF illumination at $\mathrm{pH}$ 7.4, Tfnr-phl is observed as diffuse fluorescence at the plasma membrane and as bright punctae concentrated at CCPs. When the $\mathrm{pH}$ of locally perfused buffer is switched to $\mathrm{pH}$ 5.5, Tfnr-phl at the plasma membrane and at the majority of CCPs is totally quenched. However, Tfnr-phl at a minority of clathrin coated structures remains brightly fluorescent; indicating the lumen of these structures is insulated from extracellular acidification. These structures must correspond to CCVs. By systematically switching the $\mathrm{pH}$ of locally perfused buffer between $\mathrm{pH} 7.4$ and $\mathrm{pH} 5.5$ in conjunction with image acquisition the transition of externally accessible CCPs to insulated CCVs, and hence membrane scission, can be detected at the level of single CCPs and in real time.

Using this technique in conjunction with EF/Epi microscopy we have simultaneously monitored the inward movement made by individual CCPs and membrane scission. We find that CCP movement begins well before, and culminates in, membrane scission and we thus conclude that CCP movement must correspond to CCP invagination.

\section{References}

[1] S. Mukherjee, R.N. Ghosh and F.R. Maxfield, Physiol. Rev. 77 (1997) 759.

[2] J.E. Hinshaw, Ann. Rev. Cell Dev. Biol. 16 (2000) 483.

[3] H. Stenmark, Curr. Biol. 10 (2000) R57.

[4] A.E. Engqvist-Goldstein and D.G. Drubin, Ann. Rev. Cell Dev. Biol. 19 (2003) 287.

[5] J. Rappoport, S. Simon and A. Benmerah, Traffic 5 (2004) 327.

[6] J.A. Steyer and W. Almers, Nat. Rev. Mol Cell Biol. 2 (2001) 268.

[7] D. Toomre, J.A. Steyer, P. Keller, W. Almers and K. Simons, J. Cell Biol. 149 (2000) 33.

[8] C.J. Merrifield, M.E. Feldman, L. Wan and W. Almers, Nat. Cell. Biol. 4 (2002) 691.

[9] C.J. Merrifield, B. Qualmann, M.M. Kessels and W. Almers, Eur. J. Cell Biol. 83 (2004) 13.

[10] F. Santini and J.H. Keen, Nat. Cell Biol. 4 (2002) E230. 